\title{
DER GENERATIONENROMAN ALS FIGURATION HISTORISCHER ÜBERGÄNGE. ARNO GEIGERS ES GEHT UNS GUT
}

\begin{abstract}
Generational novel as figuration of historical transitions. Arno Geiger's We Are Doing Fine Assuming that the contemporary generational novel is a kind of reflection (Widerspiegelung) of historical transitions, which is remarkable first of all on the level of commercial reception (book covers, reviews inter alia), the aim of this essay is to examine the connections between the novel's presentation of history and history itself. In the first part of the paper the terms of reflection, identity and example are proven useless to describe the problem of presentation of history in one single family's fate. Its nature is figuration - a metaphorical approach depicted by Erich Auerbach in his Mimesis in respect of the Bible and religious writings up to the Renaissance. The second part of the paper focusses on Auerbach's and his commentator's conclusions in order to uncover the figurative potential of the generational novel. Its main figure seems to be the metonymy with the assumption of pars pro toto (a part taken for the whole). Here the family metaphorically reflects the course of history through one part of its possible realizations. It differs though from the former applications by the lack of religious reference. Thus the analysis in crowned by a new "secular" allegorization of historical image in generational novels crowns.
\end{abstract}

Man erinnere sich an die Szene aus Buddenbrooks, in der die junge Tony, motiviert durch das gesellschaftliche Prestige, ihre ungewollte Verlobung eigenhändig in die häusliche Chronik einträgt und damit den fehlenden Platz jeder bürgerlichen Geschichte ausfüllt. Diese Geste, die wahrscheinlich viel mehr auf die Naivität des Mädchens als auf ihre Bereitschaft, sich selbst die Bürden des Standes aufzuerlegen, zurückzuführen ist, hat allerdings eine andere, vielmehr metaphorische Bedeutung. Es ist die Eintragung seiner selbst in den Strom der Historie, ein freiwilliges SichAnschließen an die Geschichtlichkeit, die sich in wichtigen Begebenheiten ausdrückt und dem Dokument, dem Fakt den Vorrang einräumt. Es zählt dabei das Festgelegte und das schriftlich Bekräftigte als Symptom einer Wandlung sowie als Versprechen der nach wie vor richtigen Entwicklung der Familie. Auf eine Bildlichkeit dieser Art ist der groß angelegte Generationenroman von Thomas Mann ausgerichtet. Er schildert ausführlich die Familiengeschichte in ihrer letzten Etappe - den Weg zum Verfall. In starken Streiflichtern beleuchtet er all die Ursachen des Niedergangs, die sich ankündigenden Anzeichen eines familiären Endes der Welt. 
Wie kaum ein anderes Genre orientiert sich der Generationenroman an der Versprachlichung von geschichtlichen Übergängen, schicksalsschwerer Tragik von Anfang und Ende, Dialektik des Privaten und Sozialen. ${ }^{1}$ Er beschwört die Geschichte im doppelten Sinne, verstanden erstens als ,Lauf der Welt' und deren Transformationen vor allem im Bereich der Diskurse (die sich auf geschichtliche Ereignisse übertragen), zweitens als unansehnlicher Werdegang des Phänomens Familie, der sich aus unzähligen und eigentlich unerschöpflichen Reibungen inmitten dieser kleinen sozialen Zelle zusammensetzt. ${ }^{2}$ Es stellt sich die Frage, ob das Genre ohne seinen geschichtlichen Hintergrund, ohne die Bezüge, die zu dessen Verständnis als ,Abbild der Wirklichkeit' beitragen, immer noch in reiner Form existieren, d.h. seinen Charakter bewahren könnte. Der Name selbst, der schon in sich eine Definition enthält, setzt eine Geschichtlichkeit voraus, sei es in Form der Übergänge zwischen den einzelnen Generationen, sei es durch Poetik der großen Quantifikatoren: Anfang und Ende, Vorher und Nachher, Immer und Nie.

Der letzte Popularitätsausbruch des Genres (2005), zu dessen künstlerischen und - was bemerkenswerter ist - auch kommerziellen Gipfeln Arno Geigers Es geht uns gut gehört, lässt sich wahrscheinlich durch Mechanismen der Konvention und der medialen Etablierung erläutern, die zugleich einen passenden Zeitpunkt im Bewusstsein der Leserschaft und auf dem literarischen Markt fanden. ${ }^{3}$ Was aber noch wichtiger erscheint, die Gattung wurde dem Bedürfnis der Zeit als eine Art groß angelegte Zusammenfassung des vergangenen Jahrhunderts gerecht. Dafür eignen sich wohl am besten die großen Prosaformen, die doch (wenn nicht sofort, wie die Lyrik zu ihrer engagierten Zeit) auf die historischen Umwälzungen reagieren und auf die genrespezifische Art immer noch die Aufgabe des klassischen Realismus mehr oder weniger ernst nehmen, nämlich ein Spiegel zu sein und den zeitgenössischen sowie

\footnotetext{
${ }^{1}$ Dessen ist z.B. der benachbarte Familienroman selten fähig, weil ihm eben die Idee der historischen Transformation fehlt. Er kann höchstens Generationenkonflikte darstellen, vgl. Faulkners Schall und Wahn, wo viel mehr auf Impressionen des überzeitlichen Familienschicksals als auf die Wiedergabe der geschichtlichen Entwicklung fokussiert wird. Interessanterweise wird der Generationenroman in Metzlers Literatur Lexikon noch im Jahre 2007 nicht berücksichtigt. Es wird nur von der „Generationenproblematik“ des Familienromans gesprochen (vgl. Metzler Lexikon Literatur, hrsg. von Dieter Burdorf, Christoph Fasbender, Burkhard Moennighoff, Stuttgart 2007, S. 229f.). Es scheint, dass die Unterscheidung zwischen den beiden Genres eben auf Geschichtlichkeit und Entwicklung der Familie beruhen sollte.

${ }^{2}$ Was die Produzenten von Seifenopern zu nutzen wissen, indem sie das Unerschöpfliche des Familienmotivs zur Konstruktionsregel ihrer Produkte gemacht haben.

${ }^{3}$ Dass Geigers Buch wider gängige Vorstellungen das Genre durchaus nicht wiederbelebte, geschweige denn schuf, beweisen nicht nur publizistische Aussagen (vgl. Richard Kämmerlings, http://www. welt.de/kultur/literarischewelt/article13657242/DerGenerationenroman-war-doch-nie-weg.html [24.06.2013]), sondern auch die ,vorgeigerschen' Beiträge zum Thema Generationenroman, vgl. Friederike Eigler: Gedächtnis und Geschichte in Generationenromanen seit der Wende, Berlin 2005. Zu diesem Thema auch: Anna Rutka: Erinnern und Geschlecht in zeitgenössischen deutschen Familien- und Generationenromanen, Lublin 2011, S. 45-50.
} 
künftigen Generationen als ,Reflektionsfläche' zu dienen. Was auffällt, ist gerade dieser Hang dazu, den fiktiven Stoffen die Gestalt von förmlich ,geschichtsträchtigen' Bildern zu geben und nicht zuletzt die damit verbundene Tendenz zur Etikettierung des Genres als Spiegel der Geschichte. So erzähle - gemäß der in jeder Hinsicht meinungsbildenden Buchumschläge (die wahrscheinlich einen der wirkungsvollsten rezeptionssteuernden Mechanismen darstellen) - Arno Geiger die Geschichte einer Familie, ,in deren privater Unordnung sich die politische spiegelt", weshalb er auch „ein bewegendes Stück Lebensgeschichte des 20. Jahrhunderts“ liefert. Michael Köhlmeiers Abendland steht ihm in nichts nach: „Im Spiegel zweier ungleicher Familien entsteht so ein kluger, reicher, witziger und lebenssatter Generationenroman über unsere Zeit"; ebensowenig wie auch Eugen Rüges In Zeiten des abnehmenden Lichts: „Ein halbes Jahrhundert gelebter Geschichte, ein Deutschlandroman voll überraschender Wendungen und Details“.

Diese globale historische Kennzeichnung des Erzählten muss auch nicht unbedingt in die Struktur der Werke integriert werden, sondern kann durchaus eine interpretatorische Entscheidung (von wem auch immer) sein. Man stelle sich vor, dass die ,große Geschichte', die irgendwo in den Romanen an ein paar Stellen abgestaubt wird, nur ein Bühnenbild für das wirklich Bedeutende darstellt, für die Gestaltung der Persönlichkeiten und für die Generationenkonflikte, die sich überall und immer ereignen könnten. Um nicht in die Falle der ,Universalität der Literatur' zu geraten (jeder Text ist bei näherem Hinsehen universell), lohnt es sich, eine neue Frage zu stellen: in welchem Grade, da diese ,universellen' Stories vom Werden und Sterben ans konkret Geschichtliche geheftet werden, kam dabei das ewig künstlerische Element - die Trope - abhanden? Oder aber: Inwieweit darf man von der besagten mimetischen Aufgabe sprechen, die von der herkömmlichen Konvention des Genres bedingt wird?

Der Begriff Widerspiegelung, der sich bei der Interpretation der neuen Generationenromane als nützlich zu erweisen scheint, und bei dem es sich freilich um die Widerspiegelung der politisch-gesellschaftlichen Geschichte handelt, setzt offensichtlich eine völlige Übereinstimmung voraus. Was die Romane darstellen, ist jedoch nicht die politisch-gesellschaftliche Geschichte. Es ist ein Bild der Familie, die mit der Zeit in gewisser Berührung bleibt, die einerseits von ihr geprägt wird und andererseits sie auch selbst prägt. Das Spiegelbild, von dem sich nur in Kategorien der Identität (die weder Ähnlichkeit noch Repräsentation ist) sprechen lässt, erweist sich bei dieser simplen Betrachtung als unbrauchbar - und zwar nur aus dem Grund, dass die Geschichte der Familie nicht diejenige der Welt (des Staates, der Region, der Wirtschaft, der Politik, des Diskurses usw.) ist.

Wiederum liegt die Frage nahe, ob der Generationenroman etwa nicht ein Exempel darstellt, in dem alle spezifischen Eigenheiten der Zeit (wie soziale Formung, ökonomische Verhältnisse, Denkparadigmen und Verhaltensmuster) versammelt werden. Man denke an die Anliegen des realistischen Romans, vor allem an Zola, der ein komplettes gesellschaftliches Bild seiner Epoche liefern wollte. Was ihm 
gelungen ist, stellt bis heute ein Muster an künstlerischer Gestaltung der Zeit, in der sich höchst individualisierte Charaktere in nur scheinbar exemplarischen Situationen bewegen, dar. Das Exemplarische setzt eine Regelmäßigkeit voraus, eine gewisse Abstumpfung gegen das Außerordentliche, eine Vorliebe für eine schemenhafte weil verallgemeinerte - Darstellung. Eben das ist Zola nicht gelungen - genauso wenig wie Balzac oder Flaubert. Ihre Schilderungen sind mimetisch und realistisch, aber nicht beispielhaft, weil sie das Ergebnis einer komplexen sprachlichen Gestaltung sind, die eine so weit fortgeschrittene Vereinfachung kaum rechtfertigen könnte. Da liegen die Erfahrungen mit dem sozrealistischen Roman schon näher, in dem man für die ,wahrheitstreue und historische Konkretheit" plädiert, selbst wenn seine Faszination für das Typische ihn weit außerhalb der Grenzen der Kunst platziert; man denke auch an so manches epigonale Werk, dessen (freilich rein exemplarischer) Autor das Leben , wie es ist" darzustellen vermeint. Selbstverständlich können die beiden Fälle im Kontext des hier erörterten Problems nicht ,exemplarisch' behandelt werden: ersterer ist schon längst unrühmliche Vergangenheit, der andere eignet sich wenig für die Suche nach Metaebenen.

Ein weiterer Punkt: die Figuration. Der Geschichtsbezug des Generationenromans scheint indirekt und doppeldeutig zu sein, weswegen die besagte ,Widerspiegelung' vielmehr figurale Umsetzung als genaue Ab-Schreibung bedeutet. Das Wichtigste ist, dass hier die Geschichte in den Hintergrund tritt und nicht direkt dargestellt, sondern anhand figuraler Motive angedeutet, schließlich zum Gegenstand des Erinnerungsprozesses wird, in dessen Natur die Bewältigung der Zeitlichkeit liegt. Es fragt sich, welchen Charakter, wenn überhaupt, die figurale Ebene des Genres hat und welche Verfahren genutzt werden, um sie in den Interpretationsprozess einzuführen.

Die wesentlichste, wenn nicht geradezu vollkommenste, Auslegung der literarischen Figuration liefert Erich Auerbach in Mimesis. Er interpretiert vor allem die biblischen Geschichten als figurale Sendungen, die außer ihren wörtlichen Bedeutungen Übertragung einer höheren (göttlichen) Ordnung sind, in erster Reihe Sinnbilder des Martyriums Christi. Was die antike Literatur (hier Homer als Beispiel) nicht besa $ß$ und was sie mit peinlich genauen Beschreibungen verdeckte, bietet die Bibel: innere Entwicklung der auftretenden Personen, Annäherung an das Geschichtliche, Mischung des hohen und niederen Stils, Lebensnähe, und vor allem das Imperativ einer Meta-Lektüre - erzielt durch den „Herrschaftsanspruch“4 der Bibel; entweder glaubt man an ihre Vision der Weltgeschichte als göttlicher Plan, oder man reduziert sie auf die Sage, die sich von der antiken nur durch strukturelle Merkmale unterscheidet. Entweder, lebt' man die Bibel, oder man ignoriert sie vollkommen. Ihre Eigenart befindet sich gleichsam außerhalb des wörtlichen Textes, in der institutionellen Konvention, die keinen Fehlschlag in der Deutung duldet. Außerdem ist

\footnotetext{
${ }^{4}$ Erich Auerbach: Mimesis: Dargestellte Wirklichkeit in der abendländischen Literatur, Tübingen 2001, S. 18ff.
} 
die Bibel, trotz aller Versuche, sie, weltlich' abzulesen, auch intentional doppeldeutig (die Autoren selbst glauben an die göttliche Eingebung), verweist auf eine überirdische Ordnung, auf das Jenseits, und ist nicht imstande, außerhalb dieser Auslegung ihr völliges interpretatorisches Potenzial zu bewahren. Diese Figuration muss also als Ablesen durch Tropen (vor allem Metaphern) verstanden werden, nur dass hier - im Unterschied zur populären Allegorese - die beiden Teile (das Bezeichnende und das Bezeichnete, also Figur und Erfüllung) gleichberechtigt sind. Demnach bleibt die Figur sowohl geschichtliches Gebilde als auch Verweis auf eine überzeitliche Regelmäßigkeit.

Die Interpretation per figuram hat laut Auerbach gewisse Vorteile für den mimetischen Wert des Textes. Vor allem bringt sie das Alltägliche und Private mit dem Erhabenen und Göttlichen in Einklang, sowohl auf der Handlungs- als auch auf der Stil-Ebene. Des Weiteren wird der Sage die Kraft zuerkannt, den verwickelten geschichtlichen Stoff (oder umfassender gesagt: „,ie gegenwärtige, nicht nach ästhetischen Maßstäben ausgewählte und vorgeordnete Wirklichkeit des Lebens ${ }^{\left({ }^{65}\right.}$ ) in eine Reihe von allgemein nachvollziehbaren Bildern zu integrieren. Diese Art von Figuration bewährt sich bis zur Dante-Zeit, in der sie zugleich ihren Höhepunkt und ihre Verneinung erfährt. ${ }^{6}$ Dante sperrt seine Helden in die Hölle, wo sie trotzdem historische Charaktere bleiben, die mehr am Lauf der weltlichen Dinge als am göttlichen Plan (dessen grausamen Teil sie mit ihren Qualen verwirklichen) interessiert sind. Das Jenseits verliert an Glaubwürdigkeit - es wird zum Menschen-Theater, dessen Bild sich immer stärker und konkreter durchsetzt. Dieser Bruch in der Literaturgeschichte beeinflusst die nachfolgenden Jahrhunderte. ${ }^{7}$ Nach Dante ist kaum jemand auf eine figurale Kunst fokussiert, und demzufolge: auf eine allumfassende Stilmischung, die das Hohe mit dem Niedrigen verbinden und sich sowohl der schulischen Allegorese als auch dem verflachenden Verismus entziehen könnte. An dieser Stelle jedoch verliert Auerbach sein bisheriges Interesse an der Figuration: viel mehr interessiert ihn im Weiteren die Vulgärsprache und der vierte Stand, die Garanten einer neuen realistischen Literatur werden sollen und die ihre völlige literarische Verwirklichung erst im Naturalismus finden.

Welch ein bestechender Gedanke, sich Auerbachs Figurationslehre nach der Renaissance vorzustellen! Mit der göttlichen Ordnung fehlen auch die bisherigen Bezüge. Auch die Art der Repräsentation, die sich die großen Realisten des 19. Jahrhunderts erdachten, ist anders. Ihre Schilderungen waren mimetisch aber nicht figural, weil ,das Hohe‘, als eine Möglichkeit der übertragenen Lesart, fehlte. Die

\footnotetext{
${ }^{5}$ Ebd., S. 181.

${ }^{6}$ Vgl. ebd., S. 193.

${ }^{7}$ Dagegen kann man einwenden, dass die Figuration in der Romantik immer weiterlebte. Abgesehen von einigen Versuchen, den Messianismus in ein literarisches Gewand zu kleiden (u.a. Mickiewicz in Polen), war die Epoche jedoch wenig daran interessiert, „Weltgeschichte zu erzählen“ (so Auerbach über die Bibel), und widmete sich vielmehr extrem verinnerlichten symbolischen Bildern.
} 
Wiedergabe sozialer Umstände ist noch keine Figuration. Die Figur braucht einen abstrakten Bezug, vor allem religiöser oder mythischer Natur; bei den alten Realisten ist er höchstens ethisch oder politisch. Diese Repräsentation besteht darin, dass ihre Motive nur auf eine größere Einheit und nicht auf eine andere Ebene verweisen können, wie Zolas Arbeiter auf andere Arbeiter, auf die ganze Gesellschaftsschicht, schließlich auf die sozial-ökonomische Struktur, aber nicht auf die Möglichkeit einer grundsätzlich anderen Lektüre. Eine Veränderung dieses Sachverhalts bringt das 20. Jahrhundert mit sich, die Moderne, von der Auerbach kaum Notiz nimmt, indem er den ganzen damaligen Roman mit Virginia Woolfs Psychologismus aus Zum Leuchtturm zusammenfasst.

Der polnische Übersetzer Auerbachs, Zbigniew Żabicki, fragt nach Franz Kafka, der sich nicht einmal eine Erwähnung in der Mimesis verdiente und dessen Werk doch eine Wiederbelebung der Figuration darstellt. ${ }^{8}$ Durch Symbole und Analogien entdeckt Kafka, ohne auf jegliche ,göttliche Ordnung' zu achten, überzeitliche Bezüge und Modellschicksale des Menschen; sein Verfahren ist doch die Parabel, also eine Figur. Es fehlen bei Auerbach die Namen Mann, Joyce, Brecht, Gide und ihre Varietäten der Figuration, von denen Moralität und Metaliteratur vielen wesentlichen Erscheinungen jener Zeit Ausdruck verliehen. Was Żabicki vorschlägt, ist eine Rückkehr zur figuralen Interpretation im 20. Jahrhundert, die der religiösen Bezüge schon beraubt ist, aber trotzdem auf nicht-wörtliche Dimensionen zu verweisen vermag. Der Forscher bemerkt jedoch an keiner Stelle, dass eine derartige Figuration Bestandteil jedes Textes werden kann (als Beispiel sei hier die schulische Allegorese genannt, die förmlich überall möglich wäre, wenn man nur geneigt ist, dem Text einen beliebigen didaktischen Wert zuzuschreiben). Ebenso wenig erwähnt wird die Gefahr, dass nicht jeder literarische, augenscheinlich figurale Text der Moderne dem Postulat der Geschichtlichkeit und Lebensnähe gerecht werden kann. So ist Kafka ein weniger gutes Beispiel als Thomas Mann. So nähert sich Virginia Woolf mit ihren Erinnerungslandschaften eher der Zeitlichkeit und den Versuchen, sie zu bewältigen, als James Joyce mit seinem mythologischen Intertextualitätsspiel.

Es scheint, dass das bisherige göttliche, rein religiöse Element durch andere Phänomene ersetzt werden kann, die dem Text einen figuralen Charakter gewähren. Wenn die Anforderung der Geschichtlichkeit in die Berücksichtigung der Zeit mündet, so bildet die Aufhebung der Zeit das genaue Gegenteil und zugleich eine figurale Grundlage. So war es auch in der Bibel, wo das Konkret-Menschliche immer zeitlich, seine Erfüllung dagegen - ein Platz im himmlischen Plan der Erlösung überzeitlich und nicht geschichtlich war. Eine ,verarmte', weil der religiösen Überzeitlichkeit beraubte, Variante dieses Modells vertritt eine bedeutende Strömung des gegenwärtigen Schreibens; das gilt für Proust und die von ihm beeinflusste Literatur

\footnotetext{
${ }^{8}$ Vgl. Zbigniew Żabicki: Ericha Auerbacha historia realizmu [wstęp ttumacza do pierwszego wydania polskiego], in: Erich Auerbach: Mimesis: Rzeczywistość przedstawiona w literaturze Zachodu, Warszawa 2004, S. 25.
} 
des Sich-Erinnerns, in der das Zeitliche sozusagen aus den Angeln gehoben wird; da denkt man wiederum an Virginia Woolf und wohl nicht zuletzt an Rilke. In dieser Landschaft der neuen, weltlichen' Figuration scheinen die Generationenromane des letzten Jahrzehnts immer deutlicher verankert zu sein. Was ungeklärt bleibt, ist der Charakter jener Figuration und die Basis, auf der sie entsteht.

Noch ein kurzer Blick auf die Figurationstypen, die Auerbach und seine Exegeten vorschlagen. Alle basieren auf einer Trope der Ähnlichkeit. Die frühe Figuration der Antike, des Mittelalters und der Renaissance fußt in der Allegorie. ${ }^{9}$ Ihre Eigenart bildeten eine sehr starke Konventionalisierung, Unveränderbarkeit und eine einzige Bedeutungszuschreibung. Die Metaebene der biblischen Gleichnisse war eindeutig und unabänderlich, weil hinter dem Prozess der Figuration ein ganzer, jahrhundertealter Mechanismus der Auslegung stand. So wehrte sich die Bibel erfolgreich (wie jede Art von heiliger Schrift) gegen Interpretationswillkür, weil sie durch beispiellos wirksame interpretative communities (Stanley Fish) - die institutionelle Kirche und die Gesamtheit der Gläubigen - unterstützt wurde. Auf diese Weise stimmt Dantes Werk immer noch mit der christlichen Figuration überein, selbst wenn sich das Geschichtliche und Körperliche im göttlichen Gebilde der Sphären hinterlistig durchsetzt.

Was Żabicki in seinem Kommentar zur Mimesis vorschlägt, ist das Symbol, als Zeichen der Moderne - eine willkürliche Trope. Die Autoren des vorigen Jahrhunderts, die sich schon nicht mehr um institutionalisierte Bildnisse des menschlichen Schicksals und der Geschichte zu kümmern brauchten, lieferten eine Reihe von eigenen Visionen, die sich in der Kultur etablierten. Der Name Kafkas spricht hier Bände, selbst wenn ihm die Etikette der Parabel (Allegorie im herkömmlichen Sinne) anhaftet. Der Bezug zur Geschichte, demnach zur Figuration, ist aber fraglich, und zwar wegen einer förmlich unbegrenzten Möglichkeitsfülle von Auslegungen. Dies kann auch die neue Anschauung der Geschichte verantworten, die vielmehr in nicht-stereotypen Bildern denn in fertigen Formeln vermittelt werden will. Das Symbol als mehrdeutiges, unerschöpfliches Zeichen scheint dieser Aufgabe gerecht zu werden.

Der zeitgenössische Generationenroman scheint auf einer anderen, wenngleich ebenfalls einer figuralen, Basis gegründet zu sein. Nicht auf Assoziation wie bei der Metapher (Symbol, Allegorie), sondern auf Nachbarschaft, d.h. Metonymie. Um

\footnotetext{
${ }^{9}$ Kommen wir den Vorwürfen zuvor, dass die Figuration sich wesentlich von der Allegorese unterscheide, eben durch die Gleichberechtigung der beiden Zeichenebenen, von denen die erste (die Figur) in der Allegorese vernachlässigt werde. Im obigen Fall wird nur über den Ausgangspunkt der Figuration gesprochen - die starke Konventionalisierung des Zeichens durch eine Gemeinschaft. Das scheint Auerbach selbst nahezulegen, indem er vom besagten (Anm. 4) „Herrschaftsanspruch“ der Bibel spricht, nur nimmt er wenig Rücksicht auf reader response (was freilich, im poststrukturalistischen Verständnis des Begriffs, zur Zeit der Entstehung seines Werkes nicht möglich war). Darüber hinaus spricht der Forscher auch vom Anteil der Symbole in der biblischen Figuration, er verschweigt aber, dass die ursprünglichen Symbole (wie Kreuz) sehr schnell im Rahmen der Glaubensgemeinschaft allegorisiert wurden.
} 
dies zu begründen, machen wir noch einmal einen kurzen Abstecher in Richtung Buddenbrooks.

Liest man den Roman als einen historischen, dann erweist sich der diesbezügliche Hintergrund als vage, nur ab und zu mit ziemlich knappen Mitteln angedeutet. Wenn auch etwa die Ereignisse des Jahres 1848 auf die geschäftliche Entwicklung der Familie keinen unbedeutenden Einfluss nehmen und demzufolge manch ein dramatisches Ereignis im familiären Zusammenleben hervorrufen, so ist nicht zu verhehlen, dass gerade dies eine zweit- wenn nicht drittrangige Rolle spielt. Der größte Nachdruck wird auf das Spiel der Persönlichkeiten gelegt, auf die innere Entwicklung der Protagonisten, die Bedeutsamkeit ihrer Lebensentscheidungen, schließlich auf das Fatum, den Verfall, hinter dem sicherlich ökonomische und politische Ereignisse lauern, was aber für die Auslegung des Werkes entbehrlich ist. ,Die große Geschichte" offenbart sich im Roman auf eine ganz andere Weise - erstens als gesellschaftliche Konvention, angesichts derer die Protagonisten ihre Willensstärke durchsetzen oder aber sich unterwerfen müssen, zweitens als Sprache, drittens als das eigenartige Bühnenbild, vor welchem die Handlung spielt. Das ,Universelle' des Werkes lässt sich hingegen vielmehr mythisch als Erzählung über Widrigkeiten des Schicksals, ethisch als Verwertung der Werte, ästhetisch als Durchbruch neuer Stile, philosophisch als Wechsel der Paradigmen, schließlich anthropologisch als Geschichte vom kleinen ,Ende der Welt ${ }^{`}$ lesen. ${ }^{10}$ Es stellt sich jedoch die Frage, inwieweit diese Interpretationen figural sind, und wenn schon, welche Tragkraft sie für die Lektüre des Werks besitzen. Die Weltgeschichte, die sich in äußeren Phänomenen ausdrückt, fehlt weitgehend. Die Schicht der Symbole, wenn auch reichlich vorhanden, weicht einer realistischen Familiendarstellung, wovon die individuelle, einmalige Skizzierung der Protagonisten und ihrer Eigenarten als lebendigen Menschen zeugt. Eben diese lebensnahe Schwere der Darstellung, die sich Auerbach als unverzichtbare Bedingung des mimetischen Realismus vorstellt, entkräftet die figurale Interpretation.

Geigers Es geht uns gut, das den modernen Generationenroman exemplifiziert, entwickelt sich in eine andere Richtung. Thomas Mann konzentriert sich auf die wichtigsten Ereignisse aus der Familiengeschichte, Geiger meidet sie. Mann gibt der linearen Darstellung den Vorrang, sein Nachfolger dagegen bevorzugt das sich verschachtelnde Vexierspiel der Episoden. Geigers Familiengeschichte ist hier weder beispielhaft, noch symbolisch. Es lässt sich aber nicht bestreiten, dass sie in vieler Hinsicht parallel zum Weltgeschehen verläuft. Da die Familie ein Teil eines sozialen Ganzen ist und ihre Mitglieder dabei äußerst realistisch und individualisiert in ihrem Alltag dargestellt werden, erscheint pars pro toto als die wahrscheinlichste Regel der Wirklichkeitskonstruktion. Nicht mehr das Beispielhafte und Typische, nicht mehr das Symbol als Bezug auf eine entfernte, zeitlich sowie kausal-konsekutiv locker

\footnotetext{
${ }^{10}$ Vgl. Heide Lutosch: Ende der Familie - Ende der Geschichte: Zum Familienroman bei Thomas Mann, Gabriel Garcia Márquez und Michel Houellebecq, Bielefeld 2007, S. $13 \mathrm{ff}$.
} 
verbundene Erscheinung, sondern ein Teil als Vertretung des Ganzen, in diesem Fall: die Geschichte der Familie geschildert durch ausgewählte Tage (jedes Kapitel hat ein konkretes Datum); mehr noch: die Geschichte der Welt dargestellt durch die Familiengeschichte, die sich ihrerseits in dieser Welt abspielt. Es gibt hier eine Vielfalt von möglichen metonymischen Bezügen, die die beiden Geschichtsebenen verknüpfen, somit wird versucht, sie mit den herkömmlichen Abarten der Metonymie $\mathrm{zu}$ charakterisieren. ${ }^{11}$

Auf pars pro toto stützt sich die populärste Form der Metonymie - die Synekdoche. Sie lässt sich auch am einfachsten in Bezug auf das besprochene Werk erläutern. Die im Roman Es geht uns gut dargestellte Familie Sterk/Erlach ist Zeuge wichtiger Momente in der österreichischen Historie des 20. Jahrhunderts (Anschluss, Schlacht um Wien, Unterzeichnung des Staatsvertrags). Geigers erzählerische Eigenart besteht darin, dass die Familiengeschichte jene Begebenheiten nicht vervollständigt, sondern ersetzt. Von den historischen Ereignissen erfährt der Leser indirekt, meistens über Motive, die lediglich entfernte Assoziationen daran wecken: künstliches Gebiss, Wäschegeschäft, Kanonenkugel, Fehlgeburt, Bienenzucht, Dachboden. ${ }^{12}$ Die einzelnen Episoden schildern bis auf wenige Ausnahmen (wie Peters Teilhabe an der Schlacht 1945) - alltägliche Situationen, anscheinend beliebig ausgewählte Momente des familiären Zusammenlebens. Das Wichtige wird meistens wiederum indirekt evoziert durch Erinnerungen und Assoziationen der Protagonisten, die Träger bestimmter Bedeutungen sind und die das Zurückgelegte aufs Neue erleuchten und schließlich - in die zeitliche Struktur des Erzählens hineingeschoben - jene Zeitlichkeit aufheben, durch die der veristische Realismus legitimiert wird. ${ }^{13}$ Die Weltgeschichte scheint nur angedeutet, gleichsam als Vorwand für die Familiengeschichte, die wiederum einen Teil (pars) jener Weltgeschichte bildet.

Aus dem Dickicht der ineinandergreifenden Anspielungen auf das geschichtliche Geschehen stechen solche hervor, die die Störung der kausal-konsekutiven Bezüge sowie der zeitlichen Organisation des Erzählens nutzen. Zum einen sind die Ursa-

\footnotetext{
${ }^{11}$ Meine Unterscheidung beruht ausschließlich auf den Erkenntnissen der Rhetorik, nicht auf denen der Narratologie, die den klassischen rhetorischen Begriffen eine ziemlich entfernte Bedeutung verlieh. Es muss auch gesagt werden, dass Geigers Roman die meisten Bedingungen, die die postmoderne Anwendung der Metonymie voraussetzt, vor allem die Illusionsbrechung, nicht erfüllt.

${ }^{12}$ Die meisten von diesen Motiven sind selbstverständlich Symbole, was wahrscheinlich keinen Missklang mit der metonymischen Repräsentation der Geschichte hervorruft. Als Beispiel kann Philipps Vision der Ahnenreihe als Klassenfoto fungieren - es ist freilich ein Sinnbild, eine assoziative Figur, wie die Zeichnungen von Generationen von Königen in altertümlichen Kulturen. Sie behält ihren symbolischen Charakter, selbst wenn die von ihr hervorgerufene Störung der zeitlichen Dimension als Metalepsis wirkt. Hinzuzufügen wäre auch, dass die geschichtlichen Ereignisse in wenigen Fällen durch eine Art kursiv gedruckte Presseschau direkt wiedergegeben werden.

${ }^{13}$ Vgl. die Worte des Protagonisten Philipp: „Ob man Zeit an der Hand haben kann - vergleichbar mit einem Sohn, der den Vater an der Hand nimmt und zu einem toten Tier führt": Arno Geiger: Es geht uns gut, München 2012, S. 203. Dies ist eines der vielen Sinnbilder, die eine Möglichkeit der Zeitaufhebung zulassen.
} 
chen und Folgen der geschichtlichen Veränderungen nicht bekannt; man schließt aus dem Leben der Familienmitglieder auf sie. Zum anderen ist die zeitliche Folge der einzelnen Episoden gestört, was einen (nicht unbedingt falschen) Eindruck der kausalen Umkehrung erweckt. So erfährt der Leser zuerst von dem alten Haus der Familie im Zustand des Verfalls, später erst von seiner Blütezeit; so wird einem der Tod von Ingrid eröffnet, um sie später als Kind und Mutter darzustellen; auf diese Art und Weise offenbart sich auch der Tod des Familienoberhaupts - nachdem man schon lange weiß, dass er die späteren Episoden der Handlung nicht miterleben konnte. Alles im Sinne der Metalepsis - Überschreitung der temporalen Grenzen und Ablösung der Ursache durch die Folge.

Manche der genannten Motive können in einem besonderen metonymischen Verhältnis betrachtet werden. Angefangen mit den größten - die intime Familiengeschichte als Verkleinerung der historischen Rahmenhandlung (der Staat verfällt, so auch die Familie; es kommt zum Wechsel der Denkparadigmen, so werden sie ins Generationsmosaik appliziert - alles, was man üblicherweise als ,Widerspiegelung versteht), bis zu den kleinsten (Unordnung im Haus - Unordnung der Welt; das von Peter erfundene Spiel Wer kennt Österreich? als eine Miniatur des wirklichen Staates, das zeitgleich mit ihm die Grenzen neu ziehen muss). Dieses Verfahren, das oberflächlich einer Litotes ähnelt (ohne ihre euphemistische Aussage) erreicht auch höhere Grade der Komplexität. Ihre Träger sind Fotos, Bilder und Filme, die im Roman mehrmals erwähnt und beinahe mit einer Tagebucheintragungsfunktion versehen werden. Dies sind für die (Quasi)Erinnerungsliteratur typische und schwer klassifizierbare Motive - einerseits wörtliche Widerspiegelungen, andererseits konnotativ belegte Phänomene, die ihren Charakter je nach dem Verlauf der Handlung ändern. Sie sind dabei nur anscheinend zeitlich bestimmt, da sie die Macht haben, in getarnte Erinnerungsgänge zu führen, die Gegenwart der Erzählung mitzugestalten, unverwandte Bilder heraufzubeschwören. ${ }^{14}$ Die Szenen, in denen Philipp sich die Familienfotos ansieht, oder Ingrid sich selbst als Kind im Film Hofrat Geiger ${ }^{15}$ beobachtet, eröffnen weiterreichende Perspektiven, wenn man bedenkt, dass der Leser den Protagonisten in dem Moment der Anschauung sieht. Es ist eine Teilnahme am Betrachtungsprozess der Person, die sich an den Moment des Fotografierertwerdens erinnert oder ihn sich vorstellt, wo der Schauende die Gefühle des damals Angeschauten zu entziffern versucht, indem er jetzt wiederum ihn anschaut. Diese Art der mehrmaligen Wiederholung desselben Motivs innerhalb einer Einheit, die sich wiederum in einer anderen befindet und dasselbe Motiv beinhaltet, ist auch eine Metonymie, genannt Mise-en-abyme. Sie scheint hier trotzdem ziemlich peripher zu sein, wenn auch die ihr zugrundeliegende Idee der Verkleinerung, Selbst-Referenz und Wechselbeziehung nur eines Motivs dem figuralen Verständnis verwandt ist.

\footnotetext{
${ }^{14}$ Vgl. Eigler: Gedächtnis und Geschichte, S. 163, wo den Familienfotos sogar eine die Erinnerungen ersetzende Funktion zugeschrieben wird.

${ }^{15}$ Eines der wenigen Symptome der postmodernen Metalepse als Ausgang aus der Fiktion.
} 
Die obige Klassifikation ist freilich rein strukturalistisch. Die Metonymie wurde als Prinzip bezeichnet, das die geschichtlichen Bezüge in eine Art irdische Figuration einordnet. Wichtig ist, dass jenes Prinzip noch in Buddenbrooks fehlte. ${ }^{16}$ Es scheint die Errungenschaft des neuen Generationenromans zu sein, was sich übrigens in dessen dilettantischer Lesart als ,Widerspiegelung der Zeit" unbeabsichtigt zeigt. Geigers Generationenroman legt selbst eine figurale Interpretation nahe, indem er die Weltgeschichte à rebours andeutet, indem er vom Anfang vom Ende und von der Zeit handelt, die allesamt ihre Ursprünge im religiösen Denken haben, aber durch Dialektik verweltlicht und trivialisiert wurden. Er entdeckt die andere Seite des Wandels und der Transformation, ihren übertragenen Bezug, von dem Symbole (vor allem das Motiv der lebenden Toten) und Erinnerungen zeugen. ${ }^{17} \mathrm{Zu}$ bemerken sind hier zwei widersprüchliche, aber für die Figuration typische Tendenzen: Aufhebung der Zeit und Hervorhebung der Geschichtlichkeit. Das gab es bei Mann noch nicht; die Zeit der Buddenbrooks war linear, registriert von Anfang (der Firma) an bis zu ihrem Verfall. Geiger plädiert für eine offene Struktur, in der ein Neuanfang immer möglich ist. So wäre z.B. das äußerst symbolträchtige Bild der Hausrenovierung, die Philipp unternimmt, ein figuraler Bezug auf das mythische Verständnis der Zeit als Kreis, in dem dieselben Ereignisse immer wieder zurückkehren.

Es ist noch ein anderer Aspekt der Figuration und zugleich eine gegensätzliche, weil nicht mythische, Interpretationsmöglichkeit hervorzuheben: die Protagonisten selbst können eine Figur der Geschichte sein. Als Metonymien sind sie Sklaven der Zeitlichkeit, versuchen sie zu hintergehen, stellen sich zeitlose Visionen vor, wollen die Toten wiederbeleben, pflegen Fotos, Filme, Andenken, Erinnerungen, die die vergessenen Gegenstände festhalten. ${ }^{18}$ Daraus ergibt sich das Tragische der Helden - sie wollen über die Wandlungen hinwegsehen, die ihnen die Geschichte und die Zeit im Allgemeinen aufbürdet. Jene Generationen, die sie metonymisch vertreten, entsprechen bestimmten Übergängen von Diskursen und Umbrüchen in der Geschichte. Dies lässt sich mit Hilfe von postmodernen Lebensformen nach Zygmunt Bauman erklären: Richard ist Vertreter der modernen Vorkriegsgeneration, die sich zur lebenslangen Pilgerschaft verpflichtete, Peter Teil der durch den Krieg verwüsteten Vagabundengeneration, Philipp gehört der postmodernen Touristengeneration an. ${ }^{19}$

\footnotetext{
${ }^{16} \mathrm{Zu}$ bedenken ist, dass Mann in seinem späteren Werk - in Zauberberg, noch deutlicher in Doktor Faustus - weit mehr figural ist; zugleich geschichtlich und metaphorisch.

${ }^{17}$ Virginia Woolf (Zum Leuchtturm) wäre dem ganz nahe, wenn es bei ihr einen Bezug auf Weltgeschichte gäbe. Ihre Protagonisten bewegen sich im Vakuum der Gegenwart und Vergangenheit, kreieren innere Bilder, ohne davon Notiz zu nehmen, dass sie ein Teil eines Ganzen sind.

${ }^{18}$ Vgl. die Worte der Protagonistin Alma: „Ganz ähnlich frieren die Dinge in der Erinnerung ein; als würde die Erinnerung das Farbengemisch der Vergangenheit in seine Bestandteile zerlegen und einzelne Farben herauslösen, als würde die Erinnerung die Vögel (Tauben), die vor Jahren in eilender Bewegung waren, für einen Augenblick ans Gewitter nageln“: Geiger: Es geht uns gut, S. 368.

${ }^{19}$ Vgl. Zygmunt Bauman: Ponowoczesność jako źródto cierpień, Warszawa 2000, S. 140-151.
} 
Der einzige, der die Vergangenheit loswerden kann, u.a. durch lockere Verbundenheit mit den Vorfahren, ist eben Philipp, für den die Familiengeschichte eine Art von Spiel wird ${ }^{20}$, indem er den Familienhort aufräumt, nur um ihn dann zu verlassen (wie ein typischer postmoderner Tourist). Bei ihm spielt der Zufall eine große Rolle. Er bekommt ein Haus, mit dem er wenig anfangen kann und er will die bisherige Lebensweise seiner Familie nicht fortsetzen. Da der Lauf der Generationen auf diese Art gestört und aufgehalten wird, geht an ihm die Familiengeschichte zugrunde. ${ }^{21}$ Sie verliert ihre Zeitlichkeit, hat nichts mehr zu erwarten (,Ende der Geschichte" auch im globalen Sinne). So präsentiert Geiger eine neue Version des „Verfalls einer Familie“, der sich ohne Knall und Pathos ereignet, sondern still ins Unzeitliche hinübersegelt.

Auf der Rezeptionsebene bekommt das Genre eine besondere Gestalt. Bedenken wir, dass auch Auerbach sich von konventionalisierten Lesarten leiten lässt, indem er, insbesondere im Fall der Bibel und der christlich bestimmten Literatur, vielmehr vom Verstehen der Figuration als von der Figuration selbst spricht. Eine ausschlaggebende Rolle fällt hier dem Genre zu, das sozusagen in seinem eigenen Namen gewisse Lesererwartungen kodiert. Diese Tendenz, eine Erscheinung in der Gattungsbestimmung zu versprachlichen, ist freilich nicht neu - man denke an den Briefroman als einen der großen Versuche, zuerst Gesellschaftskritik (Voltaire, Montesquieu), später Liebe (Rousseau, Goethe) zu konventionalisieren, oder aber an den Bildungs- und Entwicklungsroman, der schon im Voraus einen Werdegang des Protagonisten ankündigt und keinem anderen Ziel dienen kann. So ist auch das Beispiel der Tragödie und Komödie, wenn auch naiv, nicht abwegig: erstere beansprucht das Tragische, letztere das Komische. Es handelt sich in jedem Fall darum, dass sich schon aus dem Gattungsnamen eine Annahme allgemeiner Natur ableiten lässt, die die Gattung von vornherein einem konkreten Zweck gegenüberstellt.

So kann sich auch der Generationenroman auf einer Metaebene materialisieren, wenn sich nur eine Konvention findet, die ihn dort verfestigen kann. Dies kam auch in der Genre-Mode zum Vorschein, mit den im virtuellen Umfeld umherirrenden Interpretationen, seien es Abbild, Symbolisierung, Widerspiegelung der Geschichte. Auf diese Weise entsteht die Allegorese, auf die die Popularität des Generationenromans als geschichtliche Metonymie letzten Endes abzielt. Durch Konventionalisierung der Interpretation wird die Familie und ihr Verfall zur kanonischen Figur des Weltgeschehens, zu einer neuen Allegorie. Auf dieser Grundlage wird im medialen Raum ein Versuch unternommen, den Generationenroman als ,Bildnis der Zeit unter

\footnotetext{
${ }^{20}$ Vgl. etwa „sich Lebensläufe für Kanonekugeln ausdenken“: Geiger: Es geht uns gut, S. 101.

${ }^{21}$ Vgl. ein etwas vorsichtigeres Urteil: Joanna Ławnikowska-Koper: Polyvalentes Ich in einer hybriden Welt? Generationserfahrungen in der österreichischen Literatur der letzten Jahrhundertwende Paulus Hochgatterer, Kathrin Röggla, Arno Geiger, in: Zwischen Aufbegehren und Anpassung. Poetische Figurationen von Generationen und Generationserfahrungen in der österreichischen Literatur, hrsg. von Joanna Drynda unter Mitarbeit von Paweł Domeracki u. Marta Wimmer, Frankfurt a.M. 2012, S. 269-281, hier S. 278.
} 
dem Deckmantel einer Familiengeschichte' zu etikettieren. Der Versuch scheint trotzdem nur auf gewisse Interpretationsgemeinschaften begrenzt zu sein - man bräuchte ein ebenso wirkungsvolles Konventionalisierungsmedium wie die Bibel, um eine allgemein geltende Allegorese des ganzen Genres zu erzielen.

\section{Literatur}

Auerbach, Erich: Mimesis: Dargestellte Wirklichkeit in der abendländischen Literatur, Tübingen 2001. Bauman, Zygmunt: Ponowoczesność jako źródto cierpień, Warszawa 2000.

Eigler, Friederike: Gedächtnis und Geschichte in Generationenromanen seit der Wende, Berlin 2005.

Geiger, Arno: Es geht uns gut, München 2012.

Kämmerlings, Richard: Der Generationenroman war doch nie weg, URL: http://www.welt.de/kultur /literarischewelt/article13657242/DerGenerationenroman-war-doch-nie-weg.html [24.06.2013].

Lutosch, Heide: Ende der Familie - Ende der Geschichte: Zum Familienroman bei Thomas Mann, Gabriel García Márquez und Michel Houellebecq, Bielefeld 2007.

Ławnikowska-Koper, Joanna: Polyvalentes Ich in einer hybriden Welt? Generationserfahrungen in der österreichischen Literatur der letzten Jahrhundertwende - Paulus Hochgatterer, Kathrin Röggla, Arno Geiger, in: Zwischen Aufbegehren und Anpassung. Poetische Figurationen von Generationen und Generationserfahrungen in der österreichischen Literatur, hrsg. von Joanna Drynda unter Mitarbeit von Paweł Domeracki u. Marta Wimmer, Frankfurt a.M. 2012, S. 269-281.

Metzler Literatur Lexikon, hrsg. von Dieter Burdorf, Christoph Fasbender, Burkhard Moennighoff, Stuttgart 2007.

Rutka, Anna: Erinnern und Geschlecht in zeitgenössischen deutschen Familien- und Generationenromanen, Lublin 2011.

Żabicki, Zbigniew: Ericha Auerbacha historia realizmu (wstęp tlumacza do pierwszego wydania polskiego), in: Erich Auerbach: Mimesis: Rzeczywistość przedstawiona w literaturze Zachodu, Warszawa 2004. 